\title{
Maximal quotient rings of
}

\section{prime nonsingular group algebras}

\section{John Hannah}

Recent work by Goodearl and Handelman (see [1] and [3]) has shown that a prime, regular, right self-injective ring $Q$ must be precisely one of the following:

(a) a full linear ring (being either simple artinian or infinite dimensional full linear);

(b) a non-simple ring with zero socle;

(c) a directly finite, non-artinian ring (necessarily simple); that is, an $\mathrm{SP}(\infty)$ ring;

(d) a simple, directly infinite ring; that is, an $\mathrm{SP}(1)$ ring which is not a division ring.

Suppose now that $Q$ is the maximal right quotient ring of a (necessarily prime nonsingular) group algebra $K G$. In this thesis we try to determine how this extra hypothesis affects the above classification.

We prove in Chapter 1 that if all the conjugacy classes of $G$ are countable then $Q$ is either a full linear ring or a simple, directly infinite ring. Thus in this case only (a) and (d) of the above classification can occur.

In Chapter 2 we assume that $G$ is locally finite. Using a dimension on the finitely generated right ideals of $K G$, we show that if $G$ is a non-trivial, locally finite group with only countable conjugacy classes then $Q$ is simple and directly infinite. This is also true if $G$ is the restricted symmetric group on any infinite set.

Received 6 March 1978. Thesis submitted to the University of Canterbury, December 1977. Degree approved, February 1978. Supervisor: Dr K.C. O'Meara. 
We show in Chapter 3 that if $Q$ is a full linear ring then $G$ contains no non-trivial, locally finite, normal subgroup. If, in addition, $G$ is soluble or residually finite or $K$ has zero characteristic and $G$ is linear, then $Q$ must be simple artinian.

In Chapter 4 we generalize the above-mentioned result from Chapter 1 and, using an intersection theorem due to ZalesskiY [7], deduce that if $G$ is soluble then $Q$ must be a simple ring.

In Chapter 5 we study the ideals of $Q$ and their interaction with the normal subgroups of $G$. Our main tool here is the recent work by Goodearl and Boyle [2] on nonsingular injective modules. We show that if $G$ is residually finite then $Q$ is a simple ring.

We conclude by listing some of the questions posed by the above results.

Most of the results in the first three chapters of this thesis will appear shortly in the papers [4], [5], and [6].

\section{References}

[1] K.R. Goodearl, "Prime ideals in regular self-injective rings", Canad. J. Math. 25 (1973), 829-839.

[2] K.R. Goodearl and Ann K. Boyle, Dimension theory for nonsinguzar injective modules (Mem. Amer. Math. Soc., 177. Amer. Math. Soc., Providence, Rhode Island, 1976).

[3] K.R. Goodearl and D. Handelman, "Simple self-injective rings", Comm. AZgebra 3 (1975), 797-834.

[4] J. Hannah, "Maximal quotient rings of prime group algebras, II. Uniform right ideals", J. Austral. Math. Soc. (to appear).

[5] J. Hannah, "Quotient rings of subgroup algebras", BuZZ. London Math. Soc. (to appear).

[6] John Hannah and K.C. O'Meara, "Maximal quotient rings of prime group algebras", Proc. Amer. Math. Soc. 65 (1977), 1-7.

[7] A.E. ZalesskiY, "On the semisimplicity of a modular group algebra of a solvable group", Soviet Math. Dokl. 14 (1973), 101-105. 